ORIGINAL ARTICLE

\title{
Early diffusion weighted imaging and expression of heat shock protein 70 in newborn pigs with hypoxic ischaemic encephalopathy
}

\author{
Y Cheng, G R Liu, J T Guan, Y L Guo, Y K Li, R H Wu
}

Postgrad Med J 2005;81:589-593. doi: 10.1136/pgmi.2004.030114

See end of article for authors' affiliations

....................

Correspondence to: DrY Cheng, Department of Radiology, the Second Affiliated Hospital, Medical College of Shantou University, Shantou 515041, China; chengying662004@ yahoo.com.cn

Submitted

19 November 2004

Accepted 21 January 2005
Objective: To establish a cerebral hypoxia ischaemic model in piglets and to explore the early diffusion weighted imaging (DWI) as well as the expression of heat shock protein 70 (HSP70) with hypoxic ischaemic encephalopathy (HIE).

Methods: Twenty four 3 day old piglets were permanently ligated common carotid arteries bilaterally and ventilated with $3 \%$ oxygen for 30 minutes, and their brains were examined at three hours, six hours, and 24 hours after hypoxic ischaemia with T2 weighted images and diffusion weighted images. Apparent diffusion coefficient (ADC) values were measured and HSP70 expression was detected in the central and peripheral regions of lesions.

Results: Abnormal signal intensity was detected on DWI and ADC maps in three hypoxic ischaemic groups. ADC values in the central lesions rapidly decreased, whereas ADC values in the peripheral regions reduced prominently at 24 hours after hypoxic ischaemia. There were significant differences $(p<0.01)$ between ADC values of the central regions and the peripheral regions of lesions. The number of HSP70 positive cells was low at three hours, raised at the highest peak at six hours, and then rapidly decreased. There was statistical difference between HSP70 positive cells in the central regions and those in peripheral regions $(p<0.01)$.

Conclusion: DWI may identify more early lesions, and improve conspicuity in the acute setting. ADC values can be used as an objective measure for hypoxic ischaemic injury. Tissue reversibility can be further reflected according to the information of ADC values and HSP70 immunohistochemical staining.
$\mathrm{P}$ erinatal asphyxia remains a primary cause of hypoxic ischaemic brain injury during the neonatal period, ultimately leading to mortality or permanent neurodevelopmental sequelae in survivors. These acute changes, and the subsequent evolution of permanent brain dysfunction, have been examined in numerous investigations using both invasive and non-invasive methods. ${ }^{1-3}$ Early and accurate diagnosis is helpful not only for assessing prognosis but also for making treatment decisions. Nevertheless, clinical confirmation of ischaemic damage and prognosis are often difficult currently, when the neonates may present with nonspecific clinical features, such as seizures, hypotonia, or lethargy. ${ }^{4}$ Conventional magnetic resonance (MR) imaging techniques have been applied in both acute and chronic stages of perinatal hypoxic ischaemic encephalopathy (HIE), but they are limited in the detection of the presence and extent of hypoxic ischaemic injury attributable to incomplete myelination and the high water content of the neonatal brain. $^{5}$

Diffusion weighted imaging (DWI) has been proved to be very sensitive in diagnosing early acute cerebral ischaemia, shown capable of depicting cerebral ischaemic lesions earlier than other conventional MRI sequences in both animal and human studies, and showing regions of ischaemic insults during the hyperacute stage. ${ }^{6-11}$ But its use in detecting neonatal HIE has not been fully assessed. We examined the ability of this technique to detect cerebral changes of acute neonatal HIE in different time phases.

The apparent diffusion coefficient (ADC) is a sensitive indicator of water and ion homoeostasis of brain. Evaluation of ADC maps can improve conspicuity and calculation of ADC values can provide an objective measure for early detection of hypoxia ischaemia (HI). ${ }^{5}$ The ADC decrease occurs with reductions in cerebral blood flow that causes dysfunction of $\mathrm{Na}^{+}-\mathrm{K}^{+}$-ATPase and disruption of ionic homoeostasis. Furthermore, it might permit the definition of irreversible or potentially salvageable, reversible ischaemic damage. This abnormality in DWI and ADC is observable within minutes after onset of experimental ischaemia and occurs in human stroke before changes become apparent by 6 to 12 hours on conventional $\mathrm{T} 2$ weighted imaging. ${ }^{12}$

Expression of heat shock protein 70 (HSP70) has served as an useful indicator of cell stress in several experimental models induced by heat, excitatory stimuli, or ischaemia. ${ }^{13} 14$ The presence of the response has been correlated with neuronal survival, while the absence of the HSP 70 response in an ischaemic zone has been correlated with eventual cellular necrosis. ${ }^{15}$

This study aimed to detect the early changes of DWI, mean ADC values, and HSP70 protein expression after HI insult in newborn pigs. To test the hypothesis whether the ADC values and HSP70 expression in peripheral regions were distinct from those in the central regions at different time intervals, and whether there were potentially salvageable and reversible tissues damaged that exited in the bordering regions in the foci of HIE.

\section{METHODS}

\section{Experimental procedures}

The experimental protocols were approved by the Institutional Research Center (Shantou, China). Twenty four

Abbreviations: $A D C$, apparent diffusion coefficient; DWl, diffusion weighted imaging; HSP70, heat shock protein 70; HIE, hypoxic ischaemic encephalopathy; $\mathrm{HI}$, hypoxic ischaemia; PBS, phosphate buffered saline 

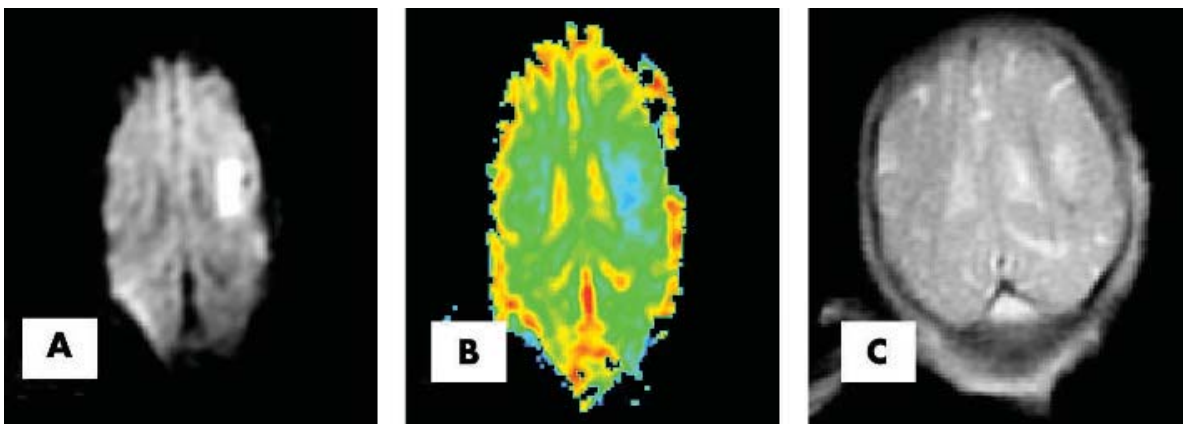

Figure 1 MRI appearance at six hours after HIE. (A) DWI hyperintensity was seen on the left temporal lobe; (B) ADC map showed considerably decreased diffusion in corresponding area; (C) T2WI hyperintensity was displayed faintly.

3 day old piglets were divided randomly into the following five groups: three in the normal control group, three in the sham operation group, six in the three hour group after HI, six in the six hour group, and six in the 24 hour group. According to the methods reported previously in the literature, ${ }^{16-18}$ the animals were anaesthetised intraperitoneally with $10 \%$ chloral hydrate $(1.5 \mathrm{ml} / \mathrm{kg})$ and placed in the supine position, a midline incision was made in the neck, then bilateral common carotid arteries were isolated and ligated permanently with a double suture. After closure of the neck wound, animals were allowed to recover from anaesthesia and were then returned to their dams. The entire surgical procedure lasted no longer than 10 minutes. About one hour after surgery, the animals were exposed to a 30 minute period of hypoxia $\left(3 \% \mathrm{O}_{2}, 97 \% \mathrm{~N}_{2}\right)$ by placing them in an airtight glass container, after which they were allowed to recover for an additional three hours, six hours, and 24 hours before MRI examination. Rectal temperature was monitored and kept at $37.2 \pm 1.3^{\circ} \mathrm{C}$ throughout the observation period.

\section{Magnetic resonance imaging}

All animals were examined using a 1.5-T MRI unit (Signa, GE Medical Systems, Milwaukee, WI). By means of a conventional surface head coil, single shot echo planar sequences were carried out for diffusion weighted imaging by varying the diffusion gradient strength along each of three orthogonal directions; diffusion weighted images (repetition time/echo time, 5000-6000 ms/112-118 ms; field of view of $20 \mathrm{~cm} \times 20 \mathrm{~cm}$; matrix of $256 \times 128$; slice thickness $5 \mathrm{~mm}$ with a zero $\mathrm{mm}$ gap) were obtained. Diffusion gradients were applied at a low b-value $\left(0 \mathrm{~s} / \mathrm{mm}^{2}\right)$ and a high b-value $\left(1000 \mathrm{~s} / \mathrm{mm}^{2}\right)$. Using commercially available software (functool) on a workstation, ADC maps were computed, and mean ADC values were calculated by drawing region of interest in central and peripheral regions of lesions. Regional evaluations of ADC values were carried out manually in three regions of interest. Fast spin echo, T2 weighted images (repetition time/echo time, $4200 / 112 \mathrm{~ms}$; field of view of $20 \mathrm{~cm} \times 20 \mathrm{~cm}$; matrix of $256 \times 192$; slice thickness, $5 \mathrm{~mm}$ with a zero $\mathrm{mm}$ gap) were obtained.

\section{HSP70 measurement}

The neonatal pigs were killed immediately after brain MRI with a lethal overdose of chloral hydrate $(450 \mathrm{mg} / \mathrm{kg})$, and then decapitated. The brains were quickly removed and soaked with $4 \%$ formalin for 24 hours, then sectioned $3 \mathrm{~mm}$ coronally from anterior to posterior, embedded in paraffin wax, finally, serial sections of $3 \mu \mathrm{m}$ in thickness were stained with haematoxylin and eosin, and streptavidin peroxidase (SP) immunoreactivity. The expression of HSP70 protein was assessed using SP immunoreactivity. Immunohistochemical staining for a neuronal marker (a mouse monoclonal IgG2a antibody against heat shock protein 70, Santa Cruz
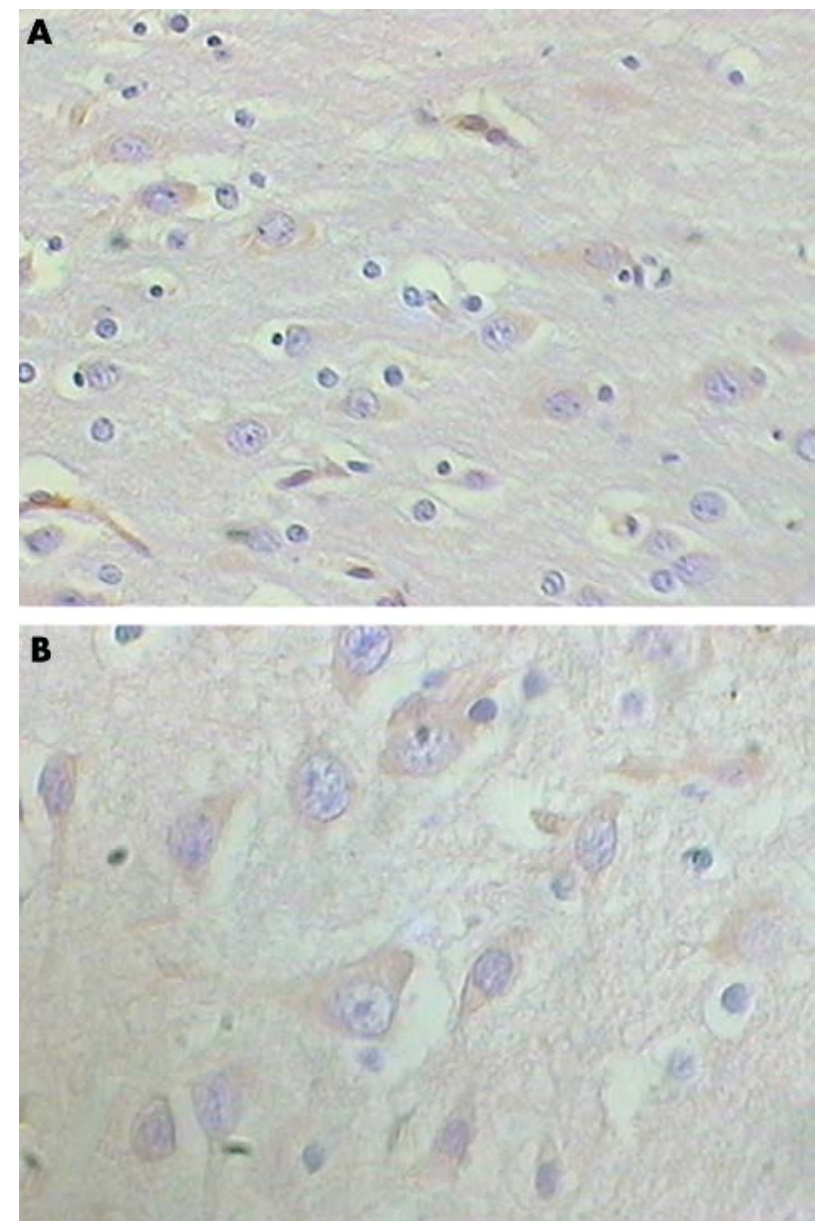

Figure 2 Immunohistochemical staining for HSP70 protein at six hours after HIE (streptavidin peroxidase stain). (A) There is brown HSP70 protein exhibited in the partial neuron cytoplasm of the central regions (original magnification $\times 200$ ). Some neurons showed pyknosis of nuclei and variance in size apparently. (B) The positive expression of HSP7O is full of the cytoplasm of neurons of the peripheral regions (original magnification $\times 400$ ). Note that neuron exists the mild abnormal appearance in the size.

Biotechnology, USA) was performed on adjacent sections according to standard protocols. Briefly, slices were treated with $3 \%$ hydrogen peroxide and $0.1 \%$ trypsin in phosphate buffered saline (PBS) for 12 minutes, and then blocked for 20 minutes with 5\% goat serum at room temperature. Dilution of 1:50 was used for the anti-HSP70 antibody; incubation with the primary antibody was performed overnight in PBS at $4^{\circ} \mathrm{C}$. Slices were rinsed for 15 minutes with PBS three times and treated for 30 minutes at $37^{\circ} \mathrm{C}$ with secondary biotinylated goat antimouse antibody (1:100, 
Santa Cruz Bio), then washed three times with PBS for five minutes each. The sections were treated with horseradish peroxidase (HRP) labelled streptavidin and stained with DAB (Santa Cruz), and then counterstained with haematoxylin and eosin. Negative controls were run in parallel and were exposed to the same steps, except that the primary antibody was eliminated. The counts of positive cells exhibiting brown HSP70 protein were measured with image analysis (HIPAS10007.0 system, China). Five autoradiographic images were analysed 200 times, and the results were averaged.

\section{Statistical analysis}

Data were analysed and graphs were designed using SigmaStat software (SPSS) 10.0 vision. Data regarding ADC values and HSP70 positive cells were given as mean (SEM). Statistical differences among groups were assessed by one way analysis of variance, and significance was determined at $\mathrm{p}$ value less than 0.05 .

\section{RESULTS \\ DWI changes}

All HI animals in this study showed areas of hyperintensity on diffusion weighted images, lesions typically located at the grey-white matter interface. Most of them were identified subcortically by focal or extensive, unilateral, or bilateral patterns. Compared with normal groups and sham operation groups, focal hyperintensity was detected clearly on diffusion weighted images at three hours after HIE; the lesions gradually extended at six hours, and became more extensive, homogeneous, or heterogeneous. At 24 hours, unilateral or bilateral hemispheres displayed diffuse hyperintensity in the brain tissues. Most lesions were homogeneous, border blurring (fig 1A).

\section{ADC measurement}

The ADC maps of all HI piglets showed hypointensity areas (fig 1B). The quantitative changes of mean ADC values that were determined by all DWI hyperintensity lesions, showed a decreasing tendency. Mean ADC values for control regions were $(1.058(0.044)) \times 10^{-3} \mathrm{~mm}^{2} / \mathrm{sc} ; \quad(1.051 \quad(0.073))$ $\times 10^{-3} \mathrm{~mm}^{2} / \mathrm{s}$. After the onset of HIE, a time dependent change of the ADC was seen. At the beginning, mean ADC in the central regions declined to $(0.556(0.065)) \times 10^{-3} \mathrm{~mm}^{2} / \mathrm{s}$ and $(0.817(0.069)) \times 10^{-3} \mathrm{~mm}^{2} / \mathrm{s}$ in the peripheral regions at three hours after HI. At about six hours, ADC continued to decrease $(0.466(0.059)) \times 10^{-3} \mathrm{~mm}^{2} / \mathrm{s}$ in the central region and $0.793(0.062)) \times 10^{-3} \mathrm{~mm}^{2} / \mathrm{s}$ in peripheral regions of lesions, then reached a minimum of 0.371 (0.023)) $\times 10^{-3} \mathrm{~mm}^{2} / \mathrm{s}$ and $\left.0.534(0.039)\right) \times 10^{-3} \mathrm{~mm}^{2} / \mathrm{s}$ at 24 hours. Mean ADC values in central regions were compared with those in peripheral regions, differences were significant $(p<0.01)$. Whereas mean ADC values in the peripheral regions between three and six hours were not significant $(p>0.05)$, those between six and 24 hours were significant $(\mathrm{p}<0.01)$.

\section{T2 weighted imaging}

No abnormal signals on T2WI were seen in the control and sham operated group, whereas the visible lesions on T2WI were noted at three hours after HI. Four of six animals showed subtle focal hyperintensity conforming to a grey and white matter junction. The hyperintensity on T2WI were significantly increased at six hours after HI, all six animals showed unilaterally more prominent hyperintensity and focal cerebral oedema. Diffuse cerebral lesions were seen in bilateral hemispheres at 24 hours after HI, including ischaemic injures, haemorrhage, and extensive swelling (fig 1C)

\section{HSP70 expression}

No HSP70 immunoreactive neurons were seen in all control and sham operated groups or in negative controls. HSP70 protein positive cells were detected as early as three hours after HIE, the number reached its peak at six hours and then declined gradually between six hours and 24 hours. The number of HSP70 positive cells were 7.36 (0.91), 8.64 (2.23), 2.64 (1.04) at three hours, six hours, and 24 hours in the central regions after the HI and 14.08 (1.96), 33.88 (2.76), 8.56 (1.67) in the peripheral regions. The greatest number of HSP70 positive neurons was seen in the peripheral area at six hours after HIE. The number of HSP70 positive cells in the central regions and the peripheral regions differed significantly in three groups $(\mathrm{p}<0.01)$ (fig $2 \mathrm{~A}$ and $\mathrm{B}$ ).

\section{DISCUSSION}

HI is a common cause of damage to the fetal and neonatal brain. The intrinsic vulnerability of specific cell types and systems in the developing brain may be more important in determining the final pattern of damage and functional disability. Focal infarction after HIE in the developing brain is a well characterised and widely used experimental marker of perinatal HI injury. This study used 3 day old piglets because at this age brain development is considered to be comparable to that of a human infant at term. ${ }^{19}$

DWI is based on the random translational movement of water molecules in biological media. The net diffusion of the molecules is called the apparent diffusion coefficient. Because the cellular structures are distributed anisotropically, the measurement of diffusion is direction dependent. Thus, to obtain a rotationally invariant estimate of the isotropic diffusion, we performed diffusion gradients in three orthogonal directions, and properly distinguished decreased diffusion with the anisotropic effect of nervous fibres. Diffusion weighting is expressed with a b value, which is dependent on sequence characteristics. The $b$ value increases with increasing DWI and the sufficient diffusion weighting is usually achieved with a b value of $1000 \mathrm{~s} / \mathrm{mm}^{2} .{ }^{20}$ In response to HI, the function of the $\mathrm{Na}^{+}-\mathrm{K}^{+}$-ATPase pump declines, leading to an influx of sodium, calcium, and water into the cell. As water in the extracellular space shows more random water diffusion than that in the restricted intracellular space, these changes in water balance result in a decrease in focal tissue diffusion. In addition, cellular swelling results in a reduction of the volume of the extracellular space. ${ }^{79^{21-23}}$ The reduced mobility of the water molecule results in hyperintensity on DWI images and hypointensity on ADC maps. Consequently, hyperintensity on DWI and decreased ADC values show the presence of cytotoxic oedema. ${ }^{7}{ }^{10}$ In our study, DWI hyperintensity occurs at three hours after HIE, greater extent of lesions are seen apparently at six hours, at 24 hours bilateral hemispheres show diffuse hyperintensity lesions, which most probably represents early injuries with cytotoxic oedema, so we postulated that the diffusion changes reflect cerebral injury developing progressively with the transition of cytotoxic oedema to extracellular oedema. ${ }^{7}$ Diffusion characteristics in the newborn brains are different from those in adults. This may be because of the neonatal distribution and regulation of cerebral blood flow, incomplete myelination, immature blood-brain barrier, and brain metabolism, resulting in that an area of decreased water diffusion is seen clearly in adults, while the same decrease will be comparatively less pronounced in the neonate. ${ }^{21-24}$

The ADC is a non-invasive, quantitative parameter of water diffusivity in brain parenchyma that is sensitive to the earliest pathophysiological changes in hyperacute ischaemic stroke. ${ }^{23}$ Defining the natural time course of ADC changes in the evolution of human stroke may provide important information for the distinction between salvageable and 
non-salvageable tissue and may be useful for monitoring therapeutic intervention. ${ }^{12}$ Differences between the water content of the neonatal and adult brain may explain some issues in neonatal ischaemia by computing ADC maps and calculating ADC values. In addition, DWI hyperintensity attributable to the $\mathrm{T} 2$ shine-through effect is removed on the $\mathrm{ADC}$ map and quantification of $\mathrm{ADC} .^{7}$ The reduction of $\mathrm{ADC}$ is attributable to disruption of energy metabolism, leading to failure of the $\mathrm{Na}^{+}-\mathrm{K}^{+}$-ATPase and other ionic pumps, leading to a loss of ionic gradients and a net translocation of water from the extracellular to the intracellular compartment. ${ }^{10}{ }^{12}$ ADC maps in this study showed hypointensity areas and reduction of $\mathrm{ADC}$ value in the central regions of lesions as early as three hours after HIE, but ADC values are normal in the peripheral regions. Similarly, six hours after HIE, the ADC values in the central regions reduced apparently, and those in the peripheral regions are still normal, reflecting the cerebral tissues in the peripheral regions of lesions are still viable within six hours, the ischaemic hyperintensity shown by DWI might be reversible if the interrupted blood flow is restored rapidly. ${ }^{22}$ With the HI injury progressively developed, ADC maps showed diffuse lesions and lower ADC values are obtained in the central and peripheral regions of lesions at 24 hours, which might be severe damaged tissues in all lesions, or irreversible tissues. In comparison with mean ADC in the peripheral regions at three time points, the mean ADC values in the central regions are lower and have significant differences $(p<0.01)$. So we conclude that brain tissue is injured more seriously in the central regions than that in the peripheral regions. The ADC value can be used as an index of lesion severity and may also be useful in distinguishing reversible from irreversible ischaemic injury. Although it is uncertain whether there is an absolute threshold of ADC reduction that predicts irreversible injury. ${ }^{5}{ }^{12}$ Decreased ADCs may be a marker of severe head injury independent of other imaging findings and clinical assessments and may be important in determining long term prognosis. ${ }^{10}$ Our results confirm that DWI signal intensity is increased and ADC is decreased at the hyperacute period (within six hours of HIE onset), whereas T2WI signal intensity is normal or mildly abnormal at three hours after HIE, this suggest no significant effect of vasogenic oedema. When T2WI hyperintensity is seen at six hours, and more prominent at 24 hours, indicating that vasogenic oedema begins to occur, and gradually worsens within the first 24 hours. ${ }^{25}$

As a stress induced protein, HSP70 exists widely in prokaryotes and eukaryotes and is the most abundant and conservative, which a neuronal self protective mechanism, renaturing the denatured proteins. However, HSP70 synthesis can be inhibited by severe ischaemic damage, it is widely accepted that expression of HSP70 immunostaining is a sensitive marker of neurons reflecting irreversible structural damage. ${ }^{26}$ This study explored the expression of HSP70 protein in neonatal pigs with cerebral HI and evaluated indirectly the tissue reversibility to forecast the prognosis with HIE. Our immunohistochemical analysis showed that induction of HSP70 was first seen at three hours after HIE, and was at a maximum level after six hours, then decreased rapidly from six hours to 24 hours in the central and peripheral regions of lesions. However, the expression of HSP70 protein was not found in the control groups. It was shown that the expression of HSP70 synthesis is very sensitive to HIE, but severe ischaemia may kill neurons without even HSP70 synthesis, suggesting the neuroprotective effect of HSP70 protein on HIE at a molecular level. ${ }^{13}{ }^{14}$ 27-30 $^{27} \mathrm{We}$ speculated that the increased neuronal function mediated by HSP70 in vivo might improve the tolerance to hypoxia. In this study, we found a high level expression of HSP70 in the peripheral neurons at six hours after HIE, suggesting that the tissue injury was mild within six hours. Thus, HSP70 may prevent the cell death or membrane injury caused by hypoxia by acting together with self protective proteins. ${ }^{4}{ }^{1427} 29$

In fact, it is impossible to detect the expression of HSP70 in vivo. Clinically, we can take advantage of the change of ADC values to reflect indirectly the injured tissues vitality. In our experiment, we found that HSP70 positive cells increased prominently with ADC values and decreased mildly to moderately within six hours after HI, showing the cerebral tissue remains vitali at that time course, if appropriate treatments were given, the injured cerebral tissue could recover entirely or partly their functions, which will produce least disability or better prognosis. But consistent with ADC decrease obviously, the expression of HSP70 rapidly decreases, even none from six hours to 24 hours after HI, we consider that the injured tissues might lose their vitality, or become irreversible, even die, even if given the appropriate treatments and it is difficult to make cerebral tissues recover from their functions, finally leading to dysfunction, or sequelae.

\section{CONCLUSION}

DWI represents a new non-invasive imaging tool and could serve as a valuable adjunct to conventional MRI. ADC maps and ADC measurements are helpful in improving the detection and depiction of extent of injury in acute HIE, and give important information for the distinction between salvageable and non-salvageable tissue. HSP70 processes neuroprotective effect with HIE and is expressed very strongly within six hours after HIE onset. Next, we will verify the reversible tissues in the peripheral regions of lesions and predict the outcome by intervention therapy.

\section{ACKNOWLEDGEMENTS}

This study is supported technically by Professor Liu and my colleagues, Yue-lin Guo and Ji-tian Guan. We thank the Medical Research Council and the clinical magnetic resonance unit for their help, and department of pathology for assistance in the immunohistochemical analysis. We are grateful for the help of Professor Wang in the preparation of the manuscript.

\section{Authors' affiliations}

Y Cheng, G R Liu, J T Guan, Y L Guo, Y K Li, R H Wu, Department of Radiology, Second Affiliated Hospital, Medical College of Shantou University, Shantou, China

Funding: none.

Conflicts of interest: none.

\section{REFERENCES}

1 Borroni GC, Schwarcz R. Neonatal asphyxia in rats: acute effects on cerebral kynurenine metabolism. Pediatr Res 2001;50:231-5.

2 Taylor DL, Mehmet H, Cady EB, et al. Improved neuroprotection with hypothermia delayed by 6 hours following cerebral hypoxia-ischemia in the 14-day-old rat. Pediatr Res 2002;51:13-19.

3 Greenwood K, Cox P, Mehmet H, et al. Magnesium sulfate treatment after transient hypoxia-ischemia in the newborn piglet does not protect against cerebral damage. Pediatr Res 2000;48:346-50.

4 Kato H, Liu XH, Nakata N, et al. Immunohistolochemical visualization of heat shock protein-70 in the gerbil hippocampus following repeated brief cerebral ischemia. Brain Res 1993;615:240-4

5 Wolf RL, Zimmerman RA, Clancy R, et al. Quantitative apparent diffusion coefficient measurements in term neonates for early detection of hypoxicischemic brain injury: initial experience. Radiology 2001;218:825-33.

6 Lin WL, Lee JM, Lee YZ, et al. Temporal relationship between apparent diffusion coefficient and absolute measurements of cerebral blood flow in acute stroke patients. Stroke 2003;34:64-70.

7 Hergan K, Schaefer PW, Sorensen AG, et al. Diffusion-weighted MRI in diffuse axonal injury of the brain. Eur Radiol 2002;12:2536-41.

8 Kawamata T, Katayama Y, Aoyama N, et al. Heterogeneous mechanisms of early edema formation in cerebral contusion: diffusion MRI and ADC mapping study. Acta Neurochir 2000;(supppl 76):9-12

9 Huisman TA, Sorensen AG, Hergan K, et al. Diffusion-weighted imaging for the evaluation of diffuse axonal injury in closed head injury. J Comput Assist Tomogr 2003;27:5-11. 
10 Chu K, Kang DW, Kim HJ, et al. Diffusion-weighted imaging abnormalities in Wernicke encephalopathy. Arch Neurol 2002;59:123-7.

11 Hill A. Current concepts of hypoxic-ischemic cerebral injury in the term newborn. Pediatr Neurol 1991;7:317-25.

12 Schlaug G, Siewert B, Benfield A, et al. Time course of the apparent diffusion coefficient (ADC) abnormality in human stroke. Neurology 1997;49:113-19.

13 Shaver EG, Welsh FA, Sutton LN, et al. Deep hypothermia diminishes the ischemic induction of heat-shock protein-72 mRNA in piglet brain. Stroke 1995; 26:1273-8.

14 Sato K, Saito H, Matsuki N. HSP70 is essential to the neuroprotective effect of heat-shock. Brain Res 1996;740:117-23.

15 Detre JA, Zager EL, Alsop DC, et al. Correlation of diffusion MRI and heat shock protein in a rat embolic stroke model. J Neurol Sci 1997; 148:163-69.

16 Park WS, Chang YS, Lee M. Effect of hypothermia on brain cell membrane function and energy metabolism after transient global hypoxia-ischemia in the newborn piglet. J Korean Med Sci 2001;16:335-41.

17 Wirrell EC, Armstrong EA, Osman LD, et al. Prolonged seizures exacerbate perinatal hypoxic-ischemic brain damage. Pediatr Res 2001;50:445-54.

18 Pulera MR, Adams LM, Liu $\mathrm{H}$, et al. Apoptosis in a neonatal rat model of cerebral hypoxia-ischemia. Stroke 1998;29:2622-30.

19 Taylor DL, Mehmet H, Cady EB, et al. Improved neuroprotection with hypothermia delayed by 6 hours following cerebral hypoxia-ischemia in the 14-day-old rat. Pediatr Res 2002;51:13-19.

20 Helenius J, Soinne L, Perkiö J, et al. Diffusion-weighted MR imaging in normal human brains in various age groups. AJNR Am J Neuroradio 2002;23:194-9.

21 Forbes KP, Pipe JG, Bird R. Neonatal hypoxic-ischemic encephalopathy: detection with diffusion-weighted MR imaging. AJNR Am J Neuroradiol 2000;21:1490-6.
22 Li FH, Liu KF, Silva $M$ et al. transient and permanent resolution of ischemic lesions on diffusion-weighted imaging after brief periods of focal ischemia in rats-correlation with histopathology. Stroke 2000;31:946-54.

23 Kuroiwa T, Nagaoka T, Ueki $M$, et al. Different apparent diffusion coefficient-water content correlation of gray and white matter during early ischemia. Stroke 1998;29:859-65.

24 Mader I, Schöning M, Klose U, et al. Neonatal cerebral infarction diagnosed by diffusion-weighted MRI pseudonormalization occurs early. Stroke 2002;33:11 142-5.

25 Robertson RL, Ben-Sira L, Bames PD, et al. MR line-scan diffusion-weighted imaging of term neonates with perinatal brain ischemia. AJNR Am J Neuroradiol 1999;20:1658-70.

26 Ringer TM, Neumann-Haefelin T, Sobel RA, et al. Reversal of early diffusionweighted magnetic resonance imaging abnormalities does not necessarily reflect tissue salvage in experimental cerebral ischemia. Stroke 2001;32:2362-9.

27 Suzuki K, Sava Y, Kaneda Y, et al. overexpressed heat shock protein 70 attenuates hypoxic injury in coronary endothelial cells. J Mol Cell Cardio 1998;30:1129-36.

28 Liu Y, Nakata KN, Kogure K. Temporal profile of heat shock protein 70 synthesis in ischemia tolerance induced by preconditioning ischemia in rat hippocampus. Neuroscience 1993;56:921-7.

29 Nishi S, Taki W, Uemura $Y$, et al. Ischemia tolerance due to the induction of HSP70 in rat ischemic recirculation model. Brain Res 1993;615:281-8.

30 Kelly S, McCulloch J, Horsburgh K. Minimal ischemic neuronal damage and HSP7O expression in MF1 strain mice following bilateral common carotid artery occlusion. Brain Res 2001;914:185-95. 